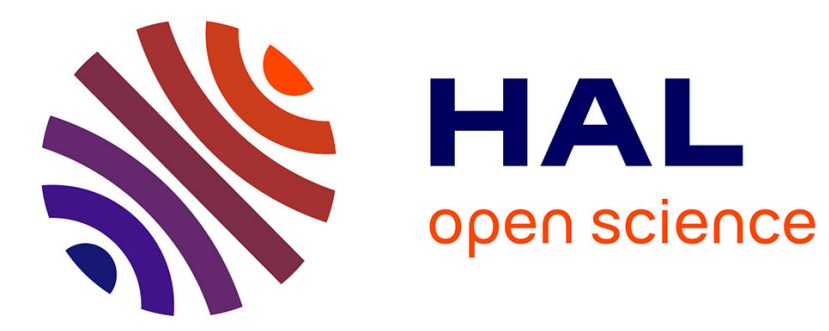

\title{
Instantaneous measurement of the intensity distribution of a focused high power laser pulse
}

\author{
L.A. Lompré, G. Mainfray, J. Thébault
}

\section{To cite this version:}

L.A. Lompré, G. Mainfray, J. Thébault. Instantaneous measurement of the intensity distribution of a focused high power laser pulse. Revue de Physique Appliquée, 1982, 17 (1), pp.21-27. 10.1051/rphysap:0198200170102100 . jpa-00244965

\section{HAL Id: jpa-00244965 https://hal.science/jpa-00244965}

Submitted on 1 Jan 1982

HAL is a multi-disciplinary open access archive for the deposit and dissemination of scientific research documents, whether they are published or not. The documents may come from teaching and research institutions in France or abroad, or from public or private research centers.
L'archive ouverte pluridisciplinaire HAL, est destinée au dépôt et à la diffusion de documents scientifiques de niveau recherche, publiés ou non, émanant des établissements d'enseignement et de recherche français ou étrangers, des laboratoires publics ou privés. 
Classification

Physics Abstracts

$07.60 \mathrm{D}-32.80 \mathrm{~K}$

\title{
Instantaneous measurement of the intensity distribution of a focused high power laser pulse
}

\author{
L. A. Lompré, G. Mainfray and J. Thébault \\ Centre d'Etudes Nucléaires de Saclay, Service de Physique des Atomes et des Surfaces, \\ 91191 Gif-sur-Yvette Cedex, France
}

(Reçu le 29 juin 1981, révisé le 21 septembre 1981, accepté le 23 septembre 1981)

\begin{abstract}
Résumé. - Un microprocesseur associé à une mémoire digitale contenant une image TV numérisée, permet de déterminer en temps réel la distribution de l'éclairement d'une impulsion laser focalisée. L'emploi d'un vidicon à mosaïque de silicium à $1,06 \mu \mathrm{m}$ ainsi que ses performances sont discutés et comparés aux résultats obtenus par des méthodes photographiques. Un système de préillumination de la cible permet de rendre parfaitement linéaire la réponse du vidicon en fonction de l'intensité incidente. La résolution de l'image numérisée est de $255 \times 255$ pixels et la dynamique du système est de 64 niveaux résolus. Cinq secondes suffisent pour calculer la section minimum $S_{\min }$ de la distribution de l'éclairement au foyer d'une lentille. En valeurs relatives, les variations de la section calculée n'excèdent pas $\pm 2 \%$, et en valeur absolue $\pm 4 \%$. Un exemple d'application de ce système est donné dans le cas de l'ionisation à 4 photons d'atomes de césium, mettant en évidence la nécessité de corriger chaque point expérimental.
\end{abstract}

\begin{abstract}
A digitalized TV system controlled by a microprocessor allows the focused intensity distribution of laser pulses to be determined instantaneously. The use of silicon mosaic vidicons at $1.06 \mu \mathrm{m}$ is discussed and their performance is compared with that obtained using photographic methods. The linearity response of the vidicon is very good provided a preillumination system is used. Dynamic ranges of 64, and $255 \times 255$ pixels are analysed. This system allows the minimum cross-section $S_{\min }$ of the focused laser intensity distribution to be measured five seconds after each laser shot, with an accuracy of $\pm 4 \%$ for absolute values, and $\pm 2 \%$ for relative variations of $S_{\min }$. The usefulness of this system is amply demonstrated in a graphical plot of the variation in the number of ions formed, as a function of laser intensity for the four-photon ionization of Cs atoms.
\end{abstract}

1. Introduction. - The measurement of the spatial profiles of laser pulses as well as their focused intensity distributions is required in most experiments concerning the interaction of laser radiation with matter. This problem becomes especially important for non linear processes such as multiphoton ionization processes, which vary with the laser intensity $I$ as $I^{N}$ [1]. A measurement of the focal-spot diameter is then often required for each laser shot. In addition, a knowledge of the absolute value of the laser intensity is necessary to make comparisons between theory and experiment.

The laser intensity is derived from the relation : $I=E / \tau S_{z}$ where $E$ is the energy of the laser pulse, $\tau$ the effective pulse duration and $S_{z}$ the effective laser cross-section in a plane $z$ which can be defined by the relation

$$
S_{z}=\int F(x, y, z) \mathrm{d} x \mathrm{~d} y
$$

$F(x, y, z)$ is the relative spatial intensity distribution in a given plane, $x, y$, are the spatial coordinates in this plane $z$.

Due to short duration of the laser pulse $\left(10^{-11} \mathrm{~s}<\tau<10^{-8} \mathrm{~s}\right)$ it is necessary to employ a method of measurement in which the instantaneous memorization of each shot is possible. Laser beam cross-section can be memorized using photographic methods. A microdensitometric treatment described in [2-4] and planimetry can be used to obtain the value of $S_{z}$, but this method takes too long for a series of shots to be evaluated in a reasonably short time. As it is often necessary to analyse the data obtained as quickly as possible in order to modify parameters during the experiment, it is necessary to calculate the value of $S_{z}$ immediately after each laser shot. The use of a computer for this calculation reduces considerably the time required; the photographic processing and necessary photometric calibration required [3] are not, however, compatible with real 
time monitoring. The present paper describes an apparatus to overcome these difficulties; it allows $S_{z}$ values to be determined only $5 \mathrm{~s}$ after the laser shot.

The system used consists of a device which serves the same purpose as the photographic plate in recording the spatial intensity distribution of the laser pulse and retains the data stored for long enough to encode $255 \times 255$ picture elements (pixels). The corresponding codes are then stored in a digital memory and subsequently processed with the aid of a low cost microprocessor. The hardware characteristics of the digital memory and the specific software used allow the value of $S_{z}$ to be calculated and displayed in $5 \mathrm{~s}$ [5]. The system can easily be coupled, if necessary, to a larger computer ; the compactness of the apparatus when used by itself is an appreciable advantage. An application of this system to multiphoton ionization of atoms is given at the end of this paper, the advantages of knowing the value of $S_{z}$ for each laser shot being demonstrated.

2. Description of the apparatus. -2.1 THE TRANSDUCER. - Silicon mosaïc vidicon transducers (SMV) are useful for recording pulsed laser images [6-7]. The sensitivity is quite good (a factor of about $10^{3}$ better than KODAK $1 \mathrm{Z}$ plates at $1.06 \mu \mathrm{m}$ ) and the input/output ratio « $\gamma$ » is equal to unity. The dynamic range that can be covered with continuous illumination exceeds 100. As with many light-sensitive devices, the dark current is temperature-dependent. The geometrical distorsion with appropriate deflexion circuitry is as low as $1 \%$. The resolution capability in continuous illumination is $625 \mathrm{TV}$ lines, corresponding to a target resolution of about 40 line pairs/mm. When the electron beam is cut-off, the target can retain the image of a short duration illumination for $40 \mathrm{~ms}$ without any appreciable loss of information, provided the temperature is not too high (less than $30^{\circ} \mathrm{C}$ ) and no parasitic light impiges on the target during the storage period. The spatial uniformity of the sensitivity is unfortunately not as good as one would like and varies, according to the devices tested, from $20 \%$ to $10 \%$ from one of the corners to the centre of the target, while spots of poor sensitivity are observed with some samples. These devices, which are good for T.V. applications, have to be carefully examined and selected if used for photometry.

Another drawback in using SMV in $1.06 \mu \mathrm{m}$ coherent light is the relative transparency of the silicon target and the quality of the input window which is not antireflection coated. Interference effects resulting in fringes on the image can occur. The number and structure of these fringes vary considerably with the tube employed and the angle of incidence of the light in relation to the target area, the least favourable conditions being a parallel beam normal to the target. An RCA SMV minimizing these effects is mentioned in [8] but this tube is not yet commercially available in Europe.
A THOMSON CSF 9826 and a RCA 4532 were selected for their qualities. The former has the best resolution when used to record short duration images, but only the latter is practically unaffected by the fringe effect and has been adopted for this reason.

2.2 IMAGE ACQUISITION. - Figure 1 shows a schematic diagram of the image acquisition method. Part of the laser beam is extracted and suitabily attenuated before being sent onto the sensitive target of the SMV, uniformly illuminated by a preillumination device $200 \mu \mathrm{s}$ before the arrival of the laser pulse. This device consists of a fast and powerful lightemitting diode LED placed $5 \mathrm{~cm}$ away from the target, outside the image field. This preillumination device was employed in order to improve the lowest flux part of the SMV response curve. A fast vacuum photodiode P.D. (RTC XA1003) supplies an electrical pulse proportional to the amplitude of the laser pulse.

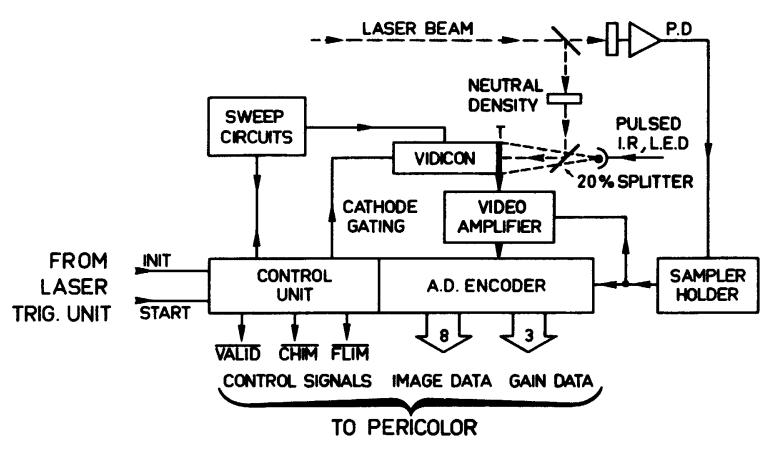

Fig. 1. - Diagram of acquisition system.

All the information such as control signals, image data and gain data, monitored by the PD electrical pulses, are fed into a digital memory and processed to give the value of $S_{z}$

Figure 2 gives the image acquisition sequence. It begins with a master reset signal which allows the data of the preceding image to be cleared from the digital memory; the system then awaits the trigger flash pulse. When this pulse occurs the electron beam of the SMV is cut-off and the preillumination begins. About $500 \mu$ s later, the laser pulse falling on the SMV and the fast diode determines the values of the gain which will be used for this particular shot. The device then awaits the first frame pulse event after the shot. This period is variable as a result of the asynchronous nature of the frame sweep. The image stored in the SMV disappears slowly; it was, however, verified that no error results from this fact. About $1.6 \mathrm{~ms}$ after the frame pulse, the digital encoding and storage begin at the rate of $540 \mathrm{~ns} /$ pixel, sending a binary value in each location of the digital memory. The computation takes place at the end of this storage sequence. It should be noted that the acquisition sequence is initiated (INIT) and triggered (START) by the laser pulse itself. 


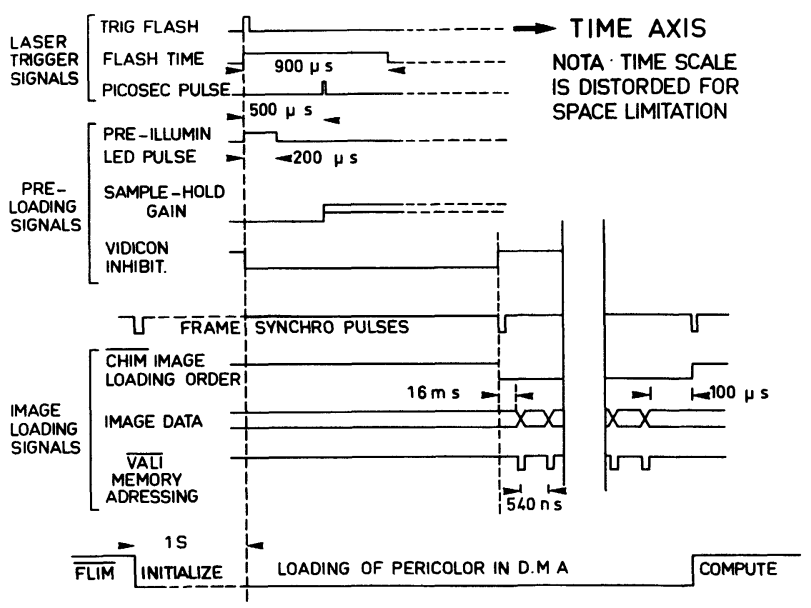

Fig. 2. - Timing diagram for image acquisition.

2.3 Video CAMERA. - Two manually switchable sweep modes using two different video amplifiers are provided. One mode is a conventional 625 line black and white standard allowing continuous display and is used for alignment. The other mode is 300 lines, 25 frames/s without overlapping and is used for digitalization. In this latter mode, the electron beam of the vidicon is controlled by the acquisition logic. The video amplifier is optimized to obtain the best dynamic range by reducing the bandwidth to the value just necessary to obtain a $255 \times 255$ pixel resolution. Provided the linearity of the SMV is assured, no gamma compensation is needed. The linearity of the video amplifier is better then $1 \%$ and the gain is controlled by the previously mentioned P.D. monitoring.

A mask is placed in front of the vidicon and the horizontal sweep adjusted to reach slightly beyond the illuminated area. This disposition allows the black level to be clamped in relation to the black pulse produced when the electron beam is out of the field, continually compensating the dark current photocathode drift.

The 8 bits, high speed digital encoder is a DATEL ADC VH8B selected for its 450 ns access time and the 8 bit-word length of its digital memory. With this arrangement the potential amplitude resolution is $1 / 255$, but unfortunately owing to imperfections in the transducer, this capacity cannot be fully employed. Particularly effective camera shielding is necessary to avoid interferences effects due to the laser high voltage supplies and R.F. sources.

2.4 Storage AND IMAGe DATA PROCESSING. 2.4.1 Digital memory. - A commercially available intelligent graphic terminal, PERICOLOR, has been interfaced with the digitalized camera (Fig. 3). PERICOLOR is a sophisticated system, which among its many possibilities, enables $64 \mathrm{~K}$ words to be sequentially addressed in the RANDOM ACCESS MEMORY (RAM). It also contains all the electro- nics necessary to display the memory content on a Colour TV Monitor after D/A conversion.

The memory characteristics chosen are :

Memory size $\quad 64 \mathrm{~K}$ words (organised $255 \times 255)$

Word Length $\quad 8$ bits +1 for cursor

Adressing MODES Random and sequential

(DIRECT MEMORY ACCESS)

Access time less than $450 \mathrm{~ns}$

Type

dynamic semiconduct. RAM

A colour-coded representation of the memory content is displayed on a colour TV Monitor immediately after the storage period and is erased only by the new content.

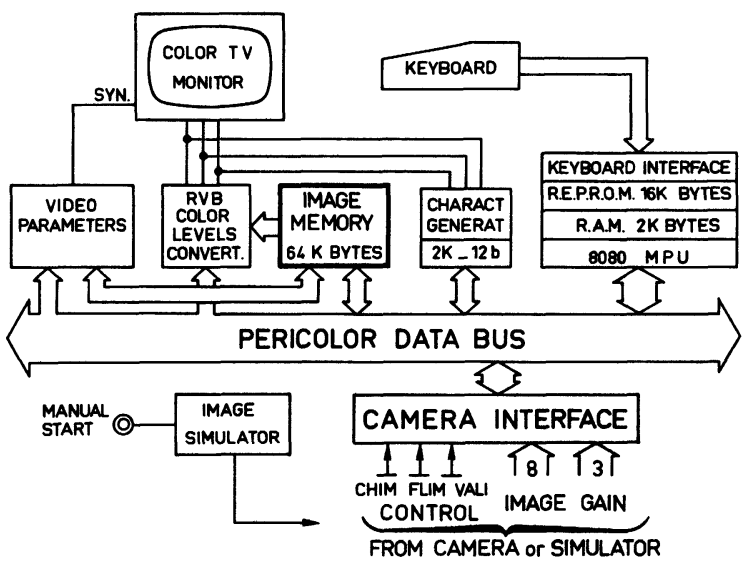

Fig. 3. - Diagram of PERICOLOR interface.

2.4.2 Microprocessor and programme. - PERICOLOR can be interfaced with a large computer; a microcomputer however is a good means of constituting an independant small-size apparatus. Furthermore, general purpose software has been developed for the 8080 microprocessor. This software very conveniently exploits the information stored in the main memory. Nevertheless, some specific assembler routines have been written : LASER SECTION and SLIT FUNCTION. These routines and the general routine are stored in $10 \mathrm{~K}$ bytes of Erasable Programmable Read Only Memory (EPROM) and can be modified as necessary. No program loading is necessary and the system starts immediately.

- LASER SECTION calculates $S_{z}$ and displays the laser cross-section value and the relative deviation with respect to a Keyboard programmable reference.

- SLIT FUNCTION displays an $x$, or $y$ curve representing the average value of the pixels contained in a dummy keyboard programmable slit, exploring the image along the $x$ or $y$ axis. With this routine the user is free to estimate the maximum and minimum memory content and to simulate the operating conditions of conventional microdensitometers. 
A joystick controlled cursor enables the experimentalist to specify the particular region of the image necessary to obtain a value of one pixel, or to obtain the average value of the pixels contained in an area encompassed by the cursor.

3. Conditions and results of tests. - $3.1 \mathrm{UNI}^{-}$ FORMITY OF THE TARGET RESPONSE. - Firstly, a particular SMV was selected by digitalizing an image of the target, uniformly illuminated by a diffused tungsten source located $2 \mathrm{~m}$ from the camera. A square mask with a $10 \mathrm{~mm}$ edge is used to limit the illuminated portion of the vidicon target. This mask is necessary for the dark current compensation mentioned above. The SLIT FUNCTION and the colour isophote mapping properties of PERICOLOR were used to examine the spatial target response of different tubes. Figure 4 shows the display on the TV monitor obtained in this test. Each point represents the average value calculated by the slit function for a given slit size. The fading corners are due to the shadow of the mask, separated from the target by the glass window of the tube. An RCA 4532 tube was finally selected. For this tube the sensitivity difference between the centre and the edge is no more than $8 \%$ (Fig. 4). The peak to peak value of high spatial frequency components (granularity) is less than $9 \%$.

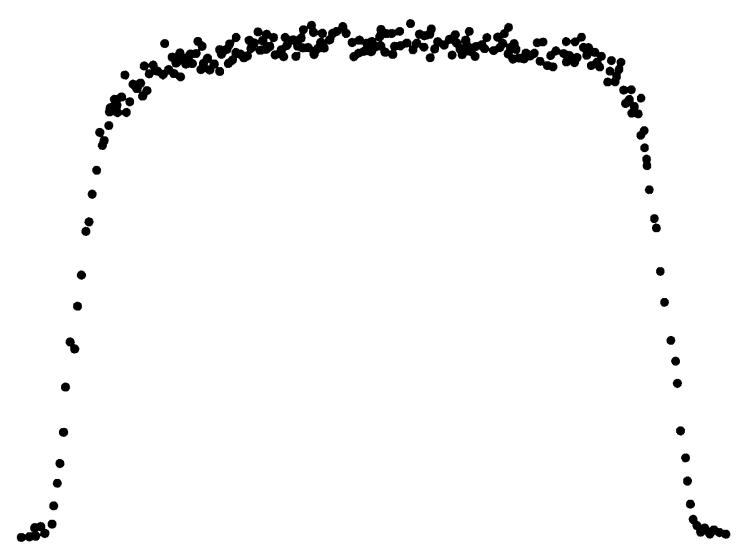

Fig. 4. - Diametral plotting of the response of a uniformly illuminated SMV target.

\subsection{TARGET RESOLUTION AND RESPONSE LINEARITY.} - After these continuous light observations some tests were performed using pulsed illumination with laser pulses. The purpose of these tests is to verify the resolution and linearity of the response under real operating conditions. To perform such an experiment, care has been taken to operate with the smallest possible number of optical elements between the light source and the SMV target in order to minimize secondary effects. For example the use of neutral densities can lead to distorsions and interference effects when coherent light is used. In order to study

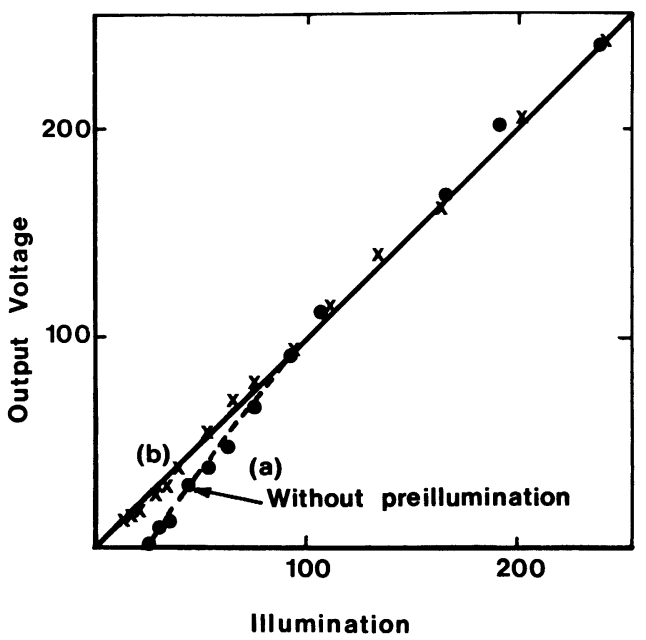

Fig. 5. - Linearity curves for the SMV illuminated by laser $: a$ ) without preillumination, $b$ ) with preillumination.

the resolution of the SMV response, a transparent resolution chart was imaged onto the SMV using a $50 \mathrm{~mm}$ focal length objective. This chart was illuminated for $30 \mathrm{ps}$ with light from a YAG modelocked $1.06 \mu \mathrm{m}$ laser diffused by ground glass.

It was found that two objects separated by $80 \mu \mathrm{m}$ on the SMV are resolved corresponding to a target resolution of 12 line pairs $/ \mathrm{mm}$.

The linearity of the response was measured by imaging, on the target, successive reflections of the laser beam between two semi-transparent mirrors with a carefully measured $75 \%$ reflection coefficient. A cylindrical lens allowed six oval spots to be obtained

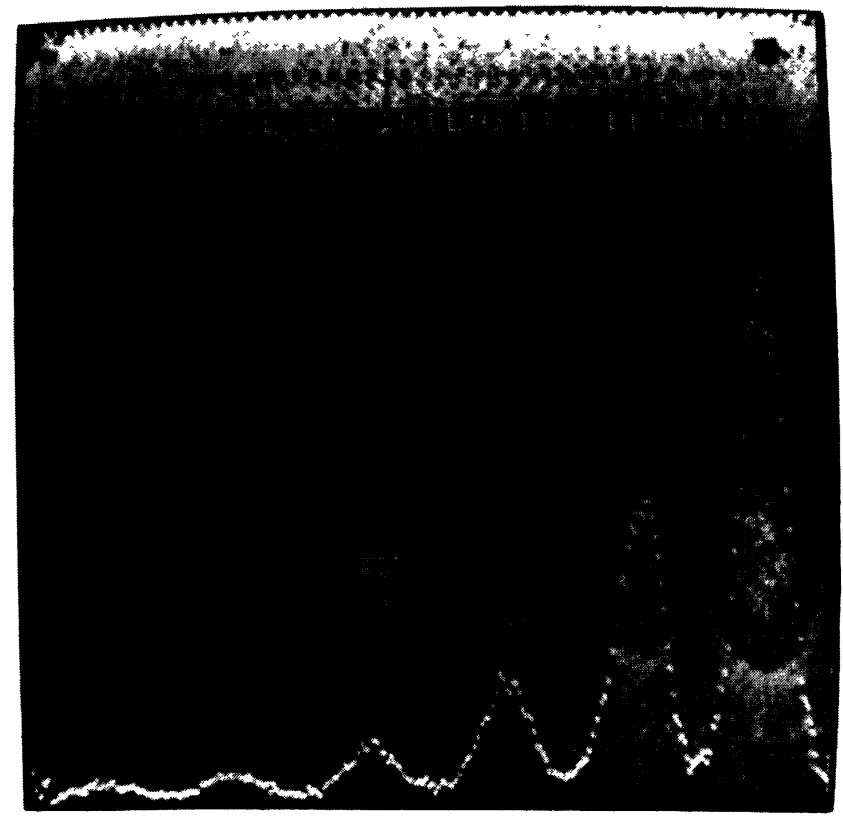

Fig. 6. - Display of successive reflections of a laser beam and plots of relative amplitudes. Six spots are clearly imaged, corresponding to 32 very significant amplitude levels, the 7th spot is off the screen but above background. 
on the target for a single laser shot. Using the SLIT FUNCTION, it is possible, as with a microdensitometer to determine the maximum value of each spot corresponding to a well defined incident intensity. Curve $5 a$ shows the response of the tube versus the relative input illumination for a $1.06 \mu \mathrm{m}$ laser with a $30 \mathrm{ps}$ pulse duration. This curve is obtained by plotting the maximum value of each spot using the slit function, as a function of the relative input illumination. This curve shows a dramatic non-linearity in the lowest flux part of the SMV response curve. This kind of non-linearity response is not suitable for computing a correct value of $S_{z}$ in the shortest possible time. To solve this problem, the preillumination device described in 2.2 part (Image acquisition) is used. Curve $5 b$ shows the effect of an extra $200 \mu$ s preillumination signal on the SMV response. It is evident that the preillumination signal improves the response of the SMV in the weak illumination part of the curve. An electrical biasing of the target was found not to produce the same result. The dynamic range obtained is illustrated in figure 6 showing successives $50 \%$ reflections of the laser beam imaged on the SMV. Using this method it was verified that the sixth spot corresponding to $1 / 64$ of the maximum intensity is at the background noise limit but nevertheless hence capable of affecting the calculated $S_{z}$ value.

3.3 Absolute $S_{z}$ value measurements. - Some tests were carried out to check the overall reproductibility of the system in the $S_{z}$ calculations. The bright area of an LED of the type used for optical fibre transmission was imagined on the SMV using $a \times 60$ microscope objective. Electrical pulses applied on the LED gave images lasting $1 \mu \mathrm{s}$ at $0.9 \mu \mathrm{m}$.

Measurements carried out on about 100 shots showed a $\pm 2 \%$ scattering of the $S_{z}$ value.

In order to evaluate the credibility of the absolute $S_{z}$ value obtained with the system, the computed $S_{z}$ value was compared with the value obtained with the photoplates/microdensitometer method. The comparison was made with a single $30 \mathrm{ps}$ laser pulse, split in order to impige on both the photoplate and the SMV.

For accurate spatial measurements of intensity, the input optics are much more critical with vidicon systems than with photographs. This is due mainly to the fact that the uniformly sensitive area of the vidicon is much smaller than the available area of the photoplate. Furthermore the photograph can be enlarged easily in non-coherent light after processing, by microdensitometer magnification, but in the vidicon the dimension of the image must be carefully adjusted under coherent light conditions. In order to make the comparison, and minimize these inconveniences, we did not use an objective lens and the half intensity diameter of the laser spot was made to cover $4 \mathrm{~mm}$ on the target of the SMV. To evaluate the absolute dimensions of this spot on the target, the shadow of a copper grid was projected onto the SMV using parallel light. Comparisons were made for 10 different shots. Each $S_{z}$ value is calculated, using the microprocesser system (5 s), and the photographic method $(\sim 10 \mathrm{~h})$. The uncertainty of the photographic method taken as reference for a given picture to analysis is $\pm 2 \%$ with a confidence level of $90 \%$. The overall uncertainty in the absolute value of $S_{z}$ measured with the apparatus is $\pm 4 \%$ provided that the dark level of the TV system and preillumination signal are correctly adjusted.

4. Applications of this apparatus. -4.1 EXPERIMENTAL PRINCIPLES. - A typical application of the system described above is given. It involves the nonresonant four-photon ionization of Cs atoms with a Nd-glass laser pulse. The $10^{9} \mathrm{~W} \cdot \mathrm{cm}^{-2}$ intensity required is obtained by focusing the laser pulse with a $50 \mathrm{~cm}$ focal length spherical lens. The experiment consists of measuring the number of ions formed as a function of the laser intensity. In most multiphoton ionization experiments, the laser intensity is varied either by inserting neutral density filters in the laser beam, or by varying the pumping power of the amplifiers. The second procedure is used in the present experiment. Both lens aberrations and laser aberrations govern the focused intensity distribution. Laser aberrations are modified by varying the laser power, and change slightly the effective diameter of the focal region [9]. Consequently, the effective diameter of the focal volume has to be measured when the laser power is varied, so that a correction has to be applied for the experimental points corresponding to the law of variation of the number of ions as a function of the laser intensity.

4.2 EXPERIMENTAL CONDITIONS. - It is necessary to magnify the small focal point with a $\times 20$ microscope objective. The calculated $S_{z}$ value corresponds to only one of the microscope objective's object planes. This simple procedure would normally be well adapted to the interaction of a laser pulse with a surface. However, it did not lead to any signyficant correlation between $S_{z}$ values and the number of ions, as preliminary results have shown. This is due to the fact that, in multiphoton ionization experiments, ions are created in a volume around the best focal point, but not in a plane. Hence it follows that the $S_{z}$ value calculated for one plane does not describe the exact value of $S_{\min }$, especially if the best focal point moves along the laser axis when the laser power is varied during the experiment. To solve this problem, several planes in the interaction volume have to be investigated simultaneously for each laser shot.

Figure 7 shows the experimental arrangement which permits the $S_{z}$ value to be calculated for 4 successive planes for each shot in the vicinity of the best focal region of the $50 \mathrm{~cm}$ focal length lens. As shown in figure 7 we use 2 parallel plane glass plates 


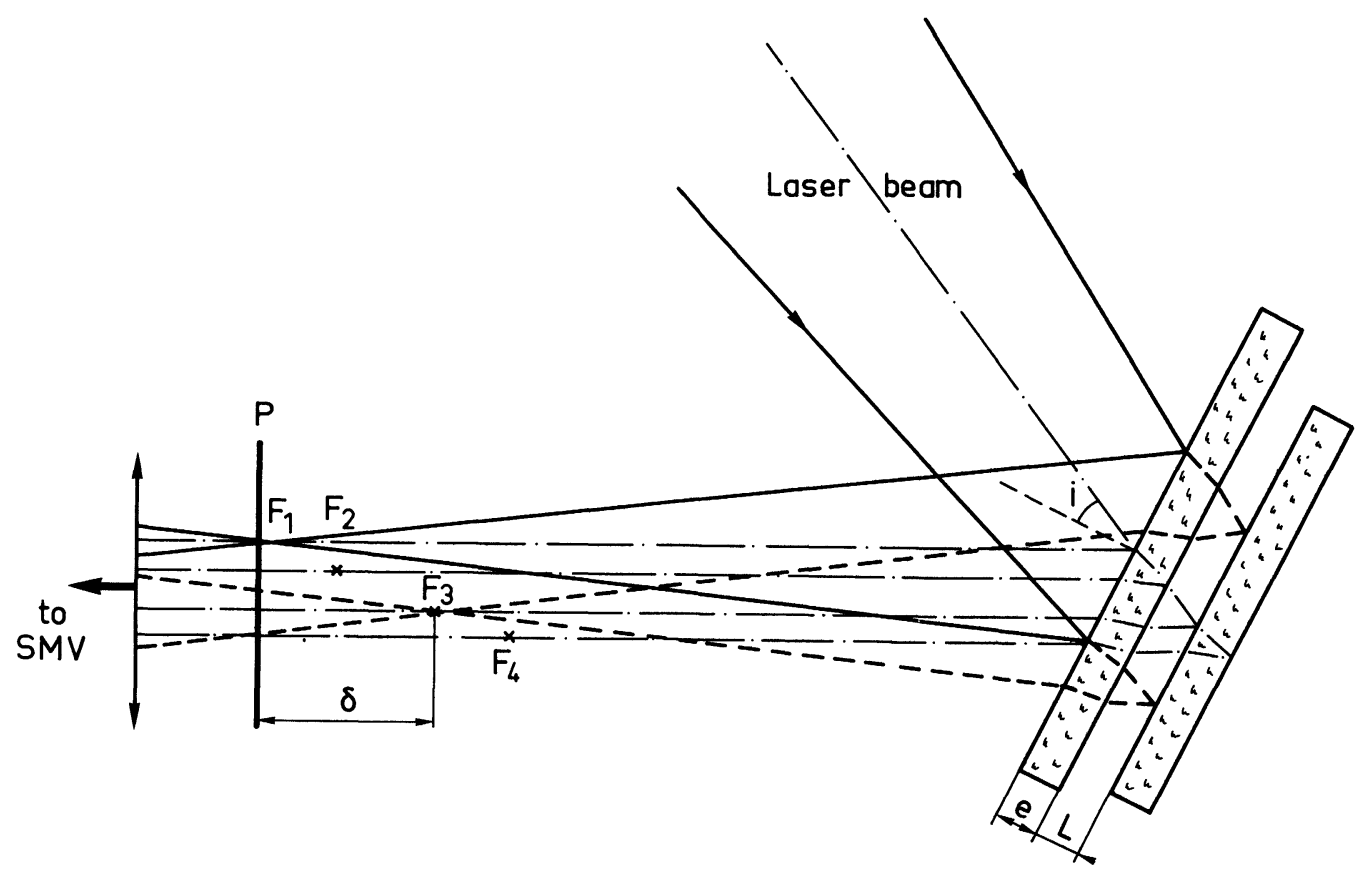

Fig. 7. - Experimental arrangement to obtain four different cross-sections of a focused laser pulse in a plane $P$.

with a thickness $e=0.75 \mathrm{~mm}$ and a refractive index $n=1.5$, separated by a distance $L=0.5 \mathrm{~mm}$. The first plate gives two reflected focused beams (focal points $F_{1}$ and $F_{2}$ ), and the second one, two further focused beams $\left(\mathrm{F}_{3}\right.$ and $\left.\mathrm{F}_{4}\right)$. The distance $\delta$ between

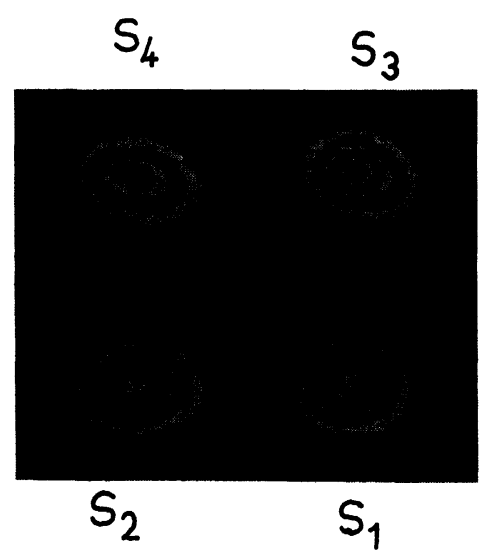

a)

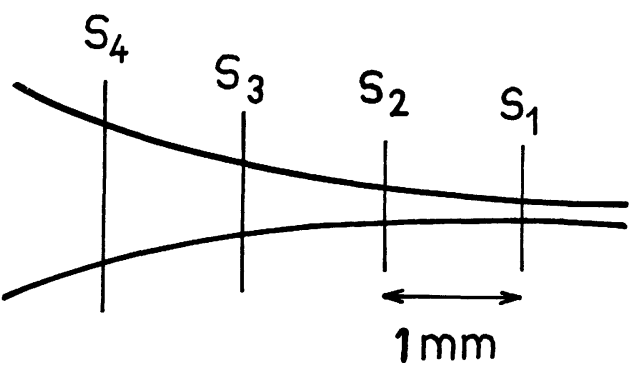

b)

Fig. 8. - a) Four isointensity contours corresponding to the laser intensity distributions in the four different planes $S_{1}, S_{2}, S_{3}$ and $S_{4}$ shown in $(b)$. one focal point, for example $F_{3}$, and the first focal point $F_{1}$ is given by

$$
\delta=2\left(\frac{e}{n}+L\right) \frac{\cos ^{2} i}{\cos r}
$$

with $n \sin r=\sin i$.

In this experiment, as $i$ is very small, the distance between two successive focal points is $1 \mathrm{~mm}$. The SMV displays 4 images from the 4 cross-sections in the plane $\mathbf{P}$ after a $\times 20$ magnification.

Figure $8 a$ shows the 4 isointensity contours obtained with the PERICOLOR system. The difference in intensity between two successive isointensity contours is $1 / 16$. The 4 isodensity contours displayed in figure $8 a$ correspond respectively to the intensity distribution in the 4 different planes $S_{1}, S_{2}, S_{3}$ and $\mathrm{S}_{4}$ shown in figure $8 b$.

4.3 EXPERIMENTAL Results. - The above described experimental arrangement has permitted the evolution of the focal volume in an experiment on four-photon ionization of $\mathrm{Cs}$ atoms to be observed visually and measured for a laser intensity variation of one order of magnitude. The law governing the variation in the number of $\mathrm{Cs}$ ions formed as a function of the laser intensity can be plotted in relative units. Figure $9 a$ shows the $\log -\log$ plot of the variation of the number of ions as a function of the laser intensity $I=E / S . \tau$ for which the focal cross-section $S$ is assumed to remain constant when the laser power is varied due to a modification in the pumping power of the amplifiers. These results give an apparent slope $K=\frac{\partial \log N i}{\partial \log I}=3.4 \pm 0.5$, with large scatter- 


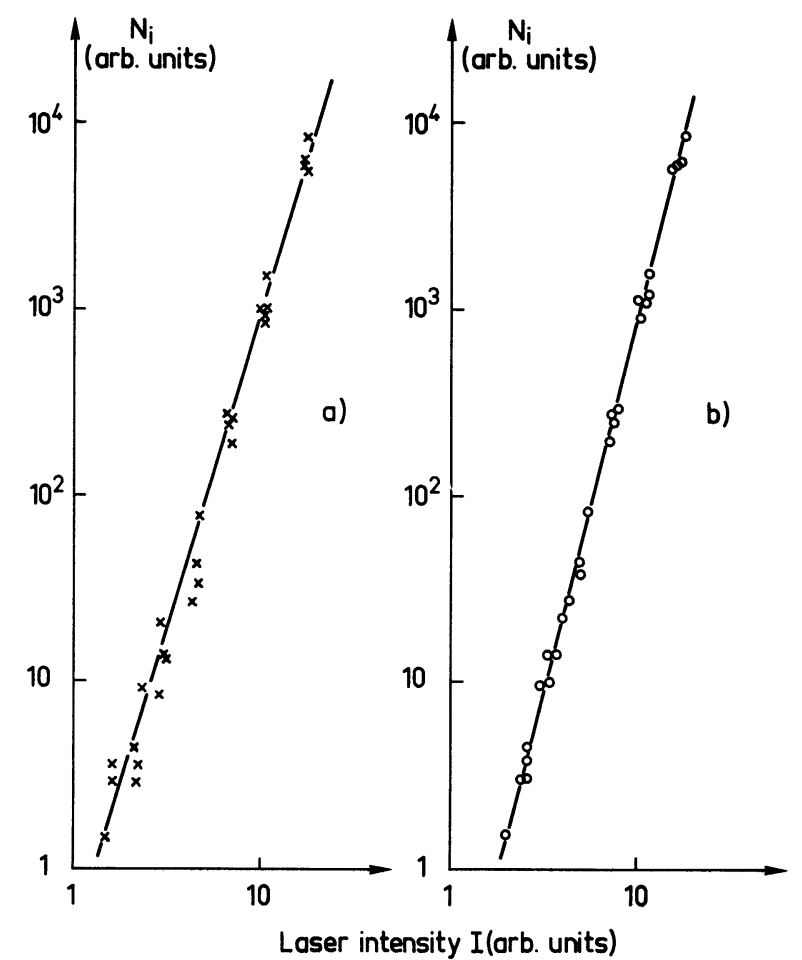

Fig. 9. - Log-log plot of the variation of the number of ions $\mathrm{Ni}$ as a function of the laser intensity $I$ in four-photon ionization of Cs atoms, $(a)$ without and $(b)$ with corrections for the focal cross-section for varying laser powers.

ing of the experimental points. The slope $K=3.4$ is inconsistent with the expected slope $K=4$ for the non-resonant four-photon ionization of $\mathrm{Cs}$ atoms. This apparent disagreement can only be explained in terms of a variation of the focal cross-section $S$ when the pumping power of the amplifiers is changed. Consequently the minimum value of the effective surface area $S_{z}$ of the focal volume has been calculated using the PERICOLOR apparatus, and the experimental arrangement described in 4.2 , for each laser shot. Figure $9 b$ shows experimental results obtained with these conditions. Here, for each experimental point, the laser intensity has been corrected to take into account the change in the effective surface area of the focal volume when the pumping power of the amplifiers is changed. A correction of up to $30 \%$ has to be applied to $S_{\min }$ when the laser intensity is varied within an order of magnitude as shown in figure $9 a$. In addition, we have measured a shift of up to $2 \mathrm{~mm}$, in the position of $S_{\min }$ along the laser axis for different laser powers. When the experimental points are corrected in this way, a curve such as that shown in figure $9 b$ is obtained. Very little scattering occurs, the results being consistent with an accurate determination of the slope

$$
K=\frac{\partial \log N i}{\partial \log I} .
$$

The slope obtained : $K=4 \pm 0.1$ is in good agreement with the expected theoretical value [2]. This example demonstrates the power of this method to obtain reliable measurements of a focused intensity distribution immediately after each laser shot.

5. Conclusion. - In conclusion, a vidicon-based method has been developed for instantaneous measurements of the focused intensity distribution of laser pulses at $1.06 \mu \mathrm{m}$. Specific qualities are a good uniformity of the spatial vidicon response, a linear response provided a preillumination is used, and a resolution of 12 line pairs $/ \mathrm{mm}$, i.e. two objects separated by $80 \mu \mathrm{m}$ on the vidicon can be resolved. Although all the tests were carried out in the near infrared, this method can also be used in the visible range.

This digitalized TV device controlled by a microprocessor was used to measure the minimum crosssection $S_{\min }$ of the focused laser intensity distribution, five seconds after individual laser shots, with an accuracy of $\pm 4 \%$ for absolute measurements, and $\pm 2 \%$ for relative variations of $S_{\min }$.

The great usefulness of this device is demonstrated in an experiment on four-photon ionization of $\mathrm{Cs}$ atoms by a laser pulse at $1.06 \mu \mathrm{m}$. It is shown that corrections of $S_{\min }$ values leading to corrections of laser intensity values give very accurate results for the variation of the number of Cs ions formed as a function of the laser intensity.

Finally, the applications of this system can be extended to instantaneous quantitative measurements of temporal and spectral laser intensity distributions, thus avoiding time-consuming photographic techniques.

\section{References}

[1] Mainfray, G. and Manus, C., J. Phys. C 1 (1978) 1.

[2] Morellec, J., Normand, D. and Petite, G., Phys. Rev. A 14 (1976) 300.

[3] Lompre, L. A., Mainfray, G., Manus, C. and TheBault, J. Phys. Rev. A 15 (1977) 1604.

[4] Normand, D. and Morellec, J., J. Phys. B 13 (1980) 1551.

[5] Lompre, L. A., Mainfray, G. and Thebault, J., Proc. of 13th Int. Congress on High speed Photography, Tokyo (1978) 558.
[6] Morellec, J., Normand, D. and Petite, G., Appl. Opt. 18 (1979) 141.

[7] Lompre, L. A., Mainfray, G. and Thebault, J., J. Appl. Phys. 48 (1977) 1570.

[8] Lee Smith, W., Degroot, A. J. and Weber, M. J., Appl. Opt. 17 (1978) 3938.

[9] Lompre, L. A., Mainfray, G., Manus, C., Repoux, S. and Thebault, J., Phys. Rev. Lett. 36 (1976) 949. 